\title{
A review of methods for assessing and managing market access and biosecurity risks using systems approaches
}

\author{
L.E. Jamieson ${ }^{1}$, H.N. DeSilva ${ }^{1}$, S.P. Worner ${ }^{2}$, D.J. Rogers ${ }^{3}$, M.G. Hill ${ }^{4}$ and J.T.S. Walker ${ }^{3}$ \\ ${ }^{1}$ The New Zealand Institute for Plant \& Food Research Limited, Private Bag 92169, \\ Auckland \\ ${ }^{2}$ Bio-Protection Research Centre, Lincoln University, PO Box 84, Canterbury, New Zealand \\ ${ }^{3}$ The New Zealand Institute for Plant \& Food Research Limited, 412 No1. Rd. \\ Te Puke, New Zealand \\ ${ }^{4}$ The New Zealand Institute for Plant \& Food Research Limited, PO Box 1401, Havelock \\ North, New Zealand \\ Corresponding author: Lisa.Jamieson@plantandfood.co.nz
}

\begin{abstract}
Systems approaches to managing risks on a pathway are increasingly seen as alternatives to single-point risk management treatments applied at the border. One key challenge of systems approaches is the difficulty in measuring risk management effectiveness across an entire pathway comprising many complex processes. The World Trade Organisation (WTO) agreement on the application of Sanitary and Phytosanitary measures (SPS) states that measures must be applied only to the extent required to achieve an appropriate degree of sanitary/phytosanitary protection (not unnecessarily traderestrictive) and must use science-based risk assessment. The framework for import risk analysis within the scope of the International Plant Protection Convention (IPPC) is outlined in the International Standards for Phytosanitary Measures (ISPMs). Although a systems approach and independent risk reduction system processes are described in various ISPMs, the methodology for determining risk across an entire pathway with complex processes is not. This contribution reviews methodologies with potential for such use.
\end{abstract}

Keywords pathways, risk analysis, Bayesian belief network, Monte Carlo simulation, data mining, machine learning, expert opinion, multiple models.

\section{INTRODUCTION}

Quarantine regulatory agencies around the world aim to "future proof" the border system to deal with long-term changes, i.e. increases in volumes of trade and passenger travel, as well as movement away from highly toxic fumigants and stricter residue restrictions on food products. Systems approaches to managing risks on import or export pathways are increasingly seen as alternatives to single-point risk management treatments applied at the border. A systems approach to managing risks on pathways is defined as "The integration of different risk management measures, at least two of which act independently, and which cumulatively 
achieve the appropriate level of protection against regulated pests" (International Standards for Phytosanitary Measures (ISPM) No. 14 (International Plant Protection Convention (IPPC) 2002)). An independent measure reduces the number or viability of pests, whereas a dependent measure (e.g. monitoring) may be required to verify efficacy of an independent measure and does not reduce the risk directly. Systems approaches can be considered when individual measures are: not adequate to meet phytosanitary import requirements; not available or likely to become unavailable; detrimental to a commodity, human health or environment; not cost effective; overly trade restrictive; and/or not feasible (Evans et al. 2012).

An overall systems approach to managing risks on an import or export pathway uses layers of biosecurity protection that take into account offshore, in-transit and on-shore activities. It involves the following processes: determining the risks at each stage of the pathway; determining the points in a pathway where risks can be reduced, monitored and controlled; establishing criteria or limits for the acceptance/failure of each independent procedure; implementing the system, with monitoring as required for the desired degree of confidence; taking corrective action when monitoring results indicate that criteria are not met; reviewing or testing to validate system efficacy and confidence on a regular basis; and maintaining adequate records and documentation (IPPC 2002).

Systems approaches provide the opportunity to consider both pre- and postharvest procedures that may contribute to the effective management of pest risk. A systems approach integrates biological, physical and operational factors that can affect the incidence, viability and reproductive potential of a pest into a system of practices and procedures that together, provide quarantine security (Jang \& Moffitt 1994). This often requires in-depth knowledge of the pest, its ecology and the impacts of the measures taken pre-and postharvest, and is therefore different from sole reliance on endpoint measures and inspection. In principle, systems approaches should be composed of the combination of phytosanitary measures that can be implemented within the exporting country in the place of production. An advantage of the systems approach is the ability to address variability and uncertainty by modifying the number and strength of measures so as to meet the appropriate degree of phytosanitary protection and confidence.

The World Trade Organisation (WTO) agreement on the application of Sanitary and Phytosanitary measures (SPS) states that measures must be applied only to the extent required to achieve the appropriate level of sanitary or phytosanitary protection (i.e. not unnecessarily trade restrictive) and must use adequate sciencebased risk assessment. Such an undertaking must include examination of the pathway from the country of origin to the destination country, the uptake in the destination country and the extent of potential exposure in the destination country. During such analysis, a quantitative or qualitative evaluation must be made of the chances of a pest occurring on the pathway (probability of entry, establishment and spread) and a corresponding quantitative or qualitative estimate of the economic consequences of biosecurity failure.

The importing country is entitled to an "appropriate level of protection" (ALOP) to guard against unwanted organisms and if the risk for entry, establishment and spread are assessed to not fall below the ALOP, the importing country can stipulate measures that the exporter must carry out in order to ensure a low risk of importing pests. Conversely, there is no entitlement to argue for zero risk. Measures chosen should be based on their effectiveness in reducing the probability of introduction of the pests, cost effectiveness and feasibility.

The risk analysis process is a technical tool that can be used to identify appropriate phytosanitary measures. It is therefore important to document the areas and degree of uncertainty in an assessment and to indicate where expert judgement has been used. This is necessary for transparency and may also be useful for identifying and prioritising research needs. Countries may decide that an appropriate degree of monitoring or audit is 
maintained to ensure that future changes in the pest risk are identified.

ISPM No. 14 is an IPPC standard for 'the use of integrated measures in a systems approach for pest risk management'. This standard provides guidelines for the development and evaluation of integrated measures in a systems approach as an option for pest risk management. Although a systems approach is described in ISPM 14, the methodology for determining risk at critical points on an entire pathway with complex processes is not.

New Zealand cannot eliminate all threats and needs to allocate resources to manage biosecurity risk, while ensuring trade and travel are maintained. Additionally, the county's export sectors aim to reduce non-tariff costs (e.g. on-shore compliance, consignment rejection, destruction or fumigation). In order to achieve such goals, a science-based transparent framework for pest risk analysis is required, using integrated measures in a systems approach that is consistent with the WTO's requirement for justifiable measures. This paper reviews those methodologies that have the potential to be used for assessing biosecurity risks.

\section{HISTORICAL DEVELOPMENT OF APPROACHES TO MANAGING BIOSECURITY RISKS}

Early approaches commonly used 'probit 9' as a criterion for treatment efficacy. Probit 9 corresponds to a mortality rate of $99.9968 \%$. However, Landolt et al. (1984) argued that probit 9 did not guarantee quarantine security because the probability of introduction depends on the number of pests surviving rather than killed and information such as pre-treatment infestation levels and other biological data are needed to calculate risks.

Baker et al. (1990) introduced the concept of Maximum Pest Limit (MPL) as a method for implementing biosecurity against quarantine pests. This limit is the smallest number of individuals capable of establishing a colony and therefore the maximum number of fruit flies (for example) that can be present in consignments imported during a specified time to a specified location. MPL is a pest compliance level used to calculate and manage risk to importers and exporters. An acceptance-sampling plan is then adopted and sample sizes calculated for a specified risk level in order to manage risk. Some pioneering work on risk assessment/management, in relation to fruit fly establishment, was carried out in New Zealand (Baker et al. 1990; Cowley et al. 1993; Maindonald 1995).

The work of Yamamura \& Katsumata (1999) focused on the concept of probability of introduction as first presented by Landolt et al. (1984). They derived equations to calculate the probability of one or more reproductive individuals passing through a port when a consignment of fruit is imported into a country. Yamamura \& Katsumata (1999) presented separate equations for four different categories of pests: sexual/parthenogenetic and solitary/gregarious. These equations allowed the initial prediction of the probability of introduction of a pest, enabling the placement of mitigating strategies through disinfestation treatments and sampling inspection to reduce the risk to an acceptable level. Further to this, Cannon (1998) pointed out the importance of risk assessment based on the probability of establishment of the pest, rather than just the probability of introduction.

Today, biosecurity is seen to be fulfilled through a more integrated approach, with biological and economic modelling of risks becoming valuable tools for the allocation of the often limited resources (Waage \& Mumford 2008). Mathematical and statistical methodologies for risk analysis are an integral part of an effective biosecurity system and some of these are discussed briefly below.

\section{METHODS FOR ANALYSING BIOSECURITY RISKS}

Conceptual models and flow charts are important in pest risk analysis, in order to capture the information and knowledge of pathways for entry, establishment and spread. Expert knowledge of the system being analysed is used to set up a conceptual model, and a graphical display of paths and inter-relationships identifies important parameters of the model. 
As a consequence of this constructing pathway diagrams to describe the risk of importation is an essential part of risk analysis process. These require knowledge of the physical systems around entry of organisms as well as the biological parameters of the pest. Assessment of risk, which follows, involves developing models through the application of statistical methods for quantifying and predicting risk, such as Bayesian Belief Networks (Bayes Nets or Probability Trees), decision networks, Monte Carlo simulations, influence diagrams, logic trees and decision trees. Expert opinion (or judgement) is an important component of such methodologies where, as is often the case, data are scarce or unavailable. Mathematical models, expert elicitation, data mining and machine learning are reviewed below.

\section{Bayesian Networks}

A Bayesian network (BN) is a probabilistic graphical model that represents a set of random variables and their conditional dependences via a directed acyclic graph (DAG). The graph consists of 'nodes' and directional arcs. The nodes represent variables, which can be discrete or continuous. The arcs show the cause-effect relationships between variables in nodes. Two variables are dependent if knowledge of one provides a predictive value for the other variable. To develop a BN, first a DAG is constructed, using expert knowledge of the system. Then, a conditional probability table (CPT), one for each child-parent node combination, is made, which gives the probability of each value of the child node conditional on every possible combination of parental node values. These can become quite complicated if the nodes have many values generally, continuous variables are combined into a few categories.

Two important elements in a BN model are marginal and conditional probabilities, and Bayes theorem. The latter is used for updating probabilities and making prediction and diagnosis. The following formula describes the Bayes rule:

$$
P\left(A_{j} \mid B\right)=\frac{P\left(B \mid A_{j}\right) \cdot P\left(A_{j}\right)}{P(B)}
$$

where $\mathrm{P}$ is probability, $\mathrm{A}$ is a set of events, $\mathrm{B}$ is another set of events and $\mathrm{j}$ is a particular outcome of the associated events.

Bayes nets are good for a visual representation of cause and effect, a platform for discussion and stakeholder input, analyses when data are scarce, combining data with expert knowledge and experience, understanding processes and testing ideas, identifying important missing data and incorporating (some elements of) uncertainty. It is best to start simply and with a narrow focus, then extend the scope later and break down the issues into sub-networks.

Kuhnert \& Hayes (2009) raised several issues that modellers need to be aware of when constructing a BN. They used an import risk assessment as one of two examples to demonstrate these issues. The authors identified discretisation, scaling, complexity and network structure as the key issues to be considered when developing a BN. Some useful advice they gave includes:

- BN software generally does not accommodate continuous probability density functions well and requires discretisation. In this situation, it is necessary to ensure that the discretisation process is an adequate representation of the underlying continuous distribution

- Network structure and complexity: Network simplicity confers some advantages, but these need to be balanced against the need for adequate description of the key processes of the system which, in turn, may add complexity. Kuhnert \& Hayes (2009) advise exploring other plausible models using influence diagrams, before deciding to parameterise a $\mathrm{BN}$. Wherever possible, consider alternative plausible models

- $\quad$ BNs are heavily reliant on expert opinion, and therefore they need to be developed through a series of workshops involving a range of experts. It is also necessary to obtain experimental data for nodes wherever possible, for validation and refinement of the model. 
'Netica' (http://www.norsys.com/) and 'GeNIe' (http://genie.sis.pitt.edu/) are popular software programmes for constructing $\mathrm{BN}$ models.

Decision networks (DN) are a generalisation of the BN, with two additional types of nodes: decision nodes and utility nodes (http://artint. info/html/ArtInt_217.html).

\section{Monte Carlo simulations}

Monte Carlo simulation is a statistical technique that is commonly used in quantitative risk analysis to simulate probabilities of input variables. In this, the uncertainty associated with each input variable is defined and modelled using a probability density function (PDF). Values for each input are randomly generated by sampling from the corresponding distribution, and these values are then used deterministically to compute the outcome of the model. The process is repeated thousands of times, generating an empirical distribution of model outcomes, which is used to provide a measure of uncertainty and probabilistic predictions of the outcome. One advantage of the Monte Carlo method compared with the $\mathrm{BN}$ approach is that there is no need to discretise continuous data if the distribution of an input variable can be approximated by a PDF. There are many theoretical PDFs with different shapes and skewness to represent the variability (and uncertainty), depending on the parameter of interest.

Leading software for Monte Carlo simulation includes Microsoft ${ }^{\circledR}$ Excel add-ins, @Risk package (http://www.palisade.com/risk/), Crystal Ball (http://crystal.software.informer.comdownloadcrystal-ball-add-in-excel/), Risk Solver (http:// www.solver.com/), Analytica (http://www.lumina. com/why-analytica/ ) and STELLA (multi-level graphical models, (http://www.iseesystems.com/ softwares/Education/StellaSoftware.aspx). A brief review of leading spreadsheet-based simulation software packages has been developed by Sugiyama (2008).

\section{Influence diagrams, logic trees and decision trees}

The objective of these methods is decisionmaking and not making probabilistic inferences, as is the case with BN and Monte Carlo methods.
An influence diagram (ID) is a simple graphical representation of a decision-making situation. The ID is a good starting point for most modelling activities. It provides an intuitive way of identifying and showing relationships between different types of nodes, i.e. decision nodes, chance variables, deterministic and objective nodes.

A decision tree is another form of graphical representation of a decision situation. It gives a chronological sequence of events that occurs in a decision situation. Again, different types of nodes can be identified. The branches from a 'Decision node' represent possible decisions, whereas those from a 'Chance node' are possible outcomes of an uncertain event or random variable, as represented by the node. Decision nodes are generally shown by rectangles and chance nodes by oval shapes. The final type of node is a terminal objective node of a set of chronologically sequenced nodes in the tree. Each of these nodes corresponds to a payoff of the combination of events that led to the node. These methods are provided in software tools available from Pallisade.com and Lumina.com.

\section{Data mining and machine learning}

Data mining is the extraction of implicit, previously unknown and potentially useful information from data. Machinelearning provides the technical basis for data mining and recently a wide range of more or less computationally intensive data mining methods have been developed (Witten et al. 2011). A number of these are finding increasing use in ecology, for example in predicting species distributions (Rodil et al. 2012) and vegetation condition (Kocev et al. 2009). Data mining methods are also beginning to be used to predict invasion risk (Keller et al. 2011; Philibert et al. 2011; Schmidt \& Drake 2011). Thus, where data exist, data mining may have value in providing estimates of pest risk. In the context of pest or disease pathway risk analysis, data sources may include pest monitoring, packhouse quality control records, spray diaries, sector pest interception records, weather data and other sources of biological data. Data mining methods may be used instead of or 
complementary to Bayesian networks to develop quantitative estimates of pest risk. Open source data mining/machine learning work benches include WEKA and MOA (Bifet et al. 2009, 2010; Witten et al. 2011).

\section{Modelling expert opinion}

Expert opinion (or judgement) is an important component of pest risk analysis where data are often scarce or unavailable. As such, expert opinions are elicited for a variety of objectives, from developing conceptual models of pest entry pathways to obtaining point estimates and uncertainties of probabilities and parameters of risk assessment models. Procedures for eliciting opinion need to be designed carefully to minimise bias. There are many factors that affect expert behaviour, and a good understanding of these is needed to design elicitation of expert opinions. Reports by Burgman et al. (2006, 2011a, 2011b), Hood et al. (2012), Speirs-bridge et al. (2010) and Martin (2012) provide good reviews of expert elicitation methods. These include following a four-step question format, with questions to obtain each expert's lowest and highest plausible value, best estimate and a degree of confidence that the true value lies between their lowest and highest value. Included is also a five-step expert elicitation approach that comprises deciding how information will be used, determining what to elicit, designing the elicitation process, performing the elicitation and encoding the elicited information for use in a model.

Methods of calibrating expert opinion are also used (Goulet et al. 2009). The calibration data dictate the influence each expert will have on the final aggregated distribution, in other words, the final estimate is weighted. It is not yet clear how such a method of weighting can be applied to expert opinion in the context of pest risk assessment. The software package $\mathrm{R}$ has a module called 'expert' that provides three different expert models for aggregation of opinion data.

Walshe \& Burgman (2010) described a framework for assessing and managing risks from emerging diseases where data are sparse, which has the potential to be applied to invasive species. The framework involves three tools that collectively comprise the means for dealing effectively with technical and social expert judgements. The tools are (1) cognitive maps to assist with topic formulation; (2) Bayes nets to describe probabilistic understanding of cause and effect; and (3) multi-criteria analysis to synthesise causal understanding and social values in a decision-making context. These three elements correspond to the stages of issue formulation, likelihood, and consequence assessment, described in a generic ecological risk analysis framework.

\section{DISCUSSION}

Management of risks using single measures can be problematic for reasons such as the measure being detrimental to the commodity, human heath and/or environment, not cost effective, not adequate to meet phytosanitary import requirements, overly trade restrictive or not feasible (Evans et al. 2012). Systems approaches offer several advantages, such as lower cost or greater robustness; however, it can be difficult, with a multifactorial system in a context of high variability, to collect the data required to formulate and negotiate a trade agreement using these methods (Whittle et al. 2012). As a result, the adoption of systems approaches to managing risks has not been as high as one would otherwise expect. However, the integrative approaches of Bayesian networks along with more quantitative mixed models offer potential to facilitate developing system approaches.

In general, the following processes are important when developing a systems approach to managing risks:

Firstly, conceptual diagrams must be constructed with input from a range of pathway users, stakeholders, regulators and researchers, to provide a structure to facilitate discussion, exchange ideas and information, identify knowledge gaps, reduce linguistic uncertainty, and to work towards a collective view of the problem. Conceptual models and flow charts are important in pest risk analysis to capture the subject matter, particularly knowledge of 
pathways for entry, establishment and spread. Expert knowledge of the system should be used to set up the conceptual model, and a graphical display of paths and inter-relationships should identify important parameters of the model. Cognitive maps (an extension of conceptual models/diagrams) can also be used to capture ideas of cause and effect and the implications of those ideas for societal or organisational values.

Secondly, there is a need to break down the complex risk analysis system into smaller submodels that deal with multiple risk factors on a small part of, or point, in the pathway. These submodels may be different from one another. They may operate independently and provide data to other sub-models in the system. Experienced modellers know that no model is perfect and that each performs differently depending on the data and the criteria used to assess its performance. Clearly, a single study or model cannot provide the breadth of information for reliable analysis of biosecurity risks.

Thirdly, the quantity of usable data available for each sub-model must be determined and, in the absence of data, decisions made about how expert judgement can be used. This leads to the use of established methods for eliciting expert opinion.

Fourthly, there is a need to synthesise empirical observations, system sub-models and expert opinion in a coherent probabilistic logical way that can be readily updated and validated. This is where using cause-and-effect models such as BNs is advantageous. As well as formulating sub-models to reflect parts of the system, future research is also required to examine how to integrate sub-models into a continuous multiple compartmental model with feedback loops to ensure that the overall system or each sub-model/models is/are validated, updated and fit for purpose.

Fifthly, in accordance with IPPC standards, there is a need to promote integrated interdisciplinary evaluation of uncertainty and validation procedures of a complex system with multiple models. Therefore, being able to represent a complex system in a simple way is also important.

As outlined in Quinlan \& Ikin (2009), the following should be considered for evaluation of a systems approach, to determine if the necessary degree of phytosanitary protection for an importing country is met: crop management practices, relevance of existing systems approach plans for comparable or identical pests on other commodities or other pests on same commodity, assessing information supplied on efficacy of measures, sampling data indicating prevalence, interception and surveillance of pest, pest-host relationships, trade costs and impact procedures for verification, appraising data alongside desired confidence levels and account options to compensate for uncertainty where necessary. Development of a systems approach requires both importing and exporting parties to provide extensive documentation at the policy level to support technical justification of any risk management measures and at the operational level to implement the risk management procedures. The approach should include a means of reporting on the performance of the systems in place.

A collaborative 'Beyond Compliance' project (Mengersen et al. 2012; Whittle et al. 2012) has been using a process for developing systems approaches referred to as "Control Point Bayesian Networks" (CP-BNs). The steps involved are: prepare a production chain for the commodity based around control points; identify and characterise measures as potential components of an systems approach; develop $\mathrm{BNs}$ for the systems approach options; analyse systems approach options; and report.

Evidence-based approaches are required to underpin the primary industry sectors' and the New Zealand Ministry for Primary Industries (MPI)'s biosecurity and market access negotiations when assessing and managing risks using systems approaches. BN models offer a good transparent platform for incorporating discrete control point data, and where datasets for risk prediction are insufficient, appropriate values can be estimated using expert elicitation methods. Where continuous data can be mined and incorporated into risk models, more quantitative methods, such as Monte Carlo simulation and detailed models for species establishment and spread, have the potential to feed outputs into a BN risk model. 


\section{ACKNOWLEDGEMENTS}

We are grateful to Richard Baker (The Food and Environmental Research Agency, UK), Mark Burgman, Stuart Jones (Australian Centre of Excellence for Risk Analysis), Naomi Cogger (Massey University), Jo Berry, Helen Harman, Mike Ormsby, Shane Olsen, Christine Reed, Shiroma Sathyapala, Melanie Newfield (The Ministry for Primary Industries), Carol Cribb, Wendy Odgers (Department of Agriculture Food \& Fishery Canberra) for attending workshops on 'Pathways Risk Analysis' and 'Assessing and Managing Risks of Biosecurity Threats Associated with Entry Pathways'. This review was supported by MBIE (Ministry of Business, Innovation, and Employment), Better Border Biosecurity (B3) (www.b3nz.org) and Market Access: Fresh Fruits (C06X0709) research programmes.

\section{REFERENCES}

Baker RT, Cowley JM, Harte DS, Frampton ER 1990. Development of a maximum pest limit for fruit flies (Diptera: Tephritidae) in produce imported into New Zealand. Journal of Economic Entomology 83(1): 13-17.

Bifet A, Kirkby R 2009. Data stream mining: a practical approach. Centre for Open Software Innovation, Waikato University. http://www.cs.waikato.ac.nz/ abifet/MOA/ StreamMining.pdf (accessed 15 May 2013).

Bifet A, Holmes G, Kirkby R Pfahringer B 2010. MOA: Massive Online Analysis. Journal of Machine Learning Research 11: 1601-1604.

Burgman M, Fidler F, McBride M, Walshe T, Wintle B. 2006. ACERA Project 0611 Eliciting Expert Judgments: Report \#1: Literature Review. ACERA, University of Melbourne, Australia. 71 p.

Burgman M, Carr A, Godden L, Gregory R, McBride M, Flander L, Maguire L 2011a. Redefining expertise and improving ecological judgment. Conservation Letters 4(2): 81-87.

Burgman MA, McBride M, Ashton R, SpeirsBridge A, Flander L, Wintle B, Fidler F, Rumpff L, Twardy C 2011b. Expert status and performance. PLoS One 6(7).
Cannon RM 1998. Sampling to comply with a maximum pest limit. Biometrics 54: 847-858. Cowley JM, Baker RT, Harte DS 1993. Measurement of parameters and application of the maximum pest limit concept for importation of hosts of tephritid fruit flies. OEPP/EPPO Bulletin 23: 713-728.

Evans H, Brokerhoff E, Baker R, Eschen R 2012. What role can the systems approach play in preventing the introduction of invasive alien species? http://hyoka.nenv.k.u-tokyo. ac.jp/alien2012/alien_report/O22_Evans.pdf (accessed 15 May 2013).

Goulet V, Jacques M, Pigeon M 2009. Expert: Modelling without data using expert opinion. Contributed Research Articles, R Journal 1/1: 31-36.

Hood G, Burgman M, Barry S 2012. Making use of expert opinion for biosecurity decisions. ACIAR Proceedings Series 137: 25-29.

International Plant Protection Convention (IPPC) 2002. The use of integrated measures in a systems approach for pest risk management. ISPM No. 14, FAO, Rome. http://www.ippc. int/file_uploaded/1146658667005_ISPM14. pdf (accessed 15 May 2013)

Jang EB, Moffitt HR 1994. Systems approaches to achieving quarantine security. In: Sharp JL, Hallman GJ ed. Quarantine treatments for pests of food plants. Westview Press Inc., Boulder, USA. Pp. 225-237.

Keller RP, Koceva D, Dzeroski S 2011. Traitbased risk assessment for invasive species: high performance across diverse taxonomic groups, geographical ranges and machine learning/statistical tools. Diversity and Distributions 17: 451-461.

Kocev D, Dzeroski S, White MD, Newell GR, Griffioen P 2009. Using single- and multitarget regression trees and ensembles to model a compound index of vegetation condition. Ecological Modelling 220: 1159-1168.

Kuhnert PM, Hayes KR 2009. How believable is your BBN? Proceedings of the 18th World AMACS / MODSIM Congress. 13-17 July, Cairns, Australia. http://mssanz.org.au/modsim09/J3/kuhnert_ J3.pdf (accessed 6 June 2013). Pp. 4319-4325. 
Landolt PJ, Chambers DL, Chew V 1984. Alternative to the use of probit 9 mortality as a criterion for quarantine treatments of fruit fly (Diptera: Tephritidae) -infested fruit. Journal of Economic Entomology 77: 285-287.

Maindonald JH 1995. The challenges of pest risk analysis. In: Bartlett PW, Chaplin GR, van Velsen R ed. Plant Quarantine Statistics. A Review. Proceedings of an international workshop, Sydney, Australia. Horticulture and Research Development Corporation, Gordon, Australia.

Martin T, Burgman M, Fidler F, Kuhnert P, Low-Choy S, MacBride M, Mengersen K 2012. Elicitation of expert knowledge in conservation biology. Conservation Biology 26(1): 29-38.

Mengersen K, Quinlan MM, Whittle PJL, Knight JD, Mumford JD, Wan Ismail WN, Tahir H, Holt J, Leach AW, Johnson S, Sivapragasam A, Lum KY, Sue MJ, Othman Y, Jumaiyah L, Tu DM, Anh NT, Pradyabumrung T, Salyapongse C, Marasigan LQ, Palacpac MB, Dulce L, Panganiban GGF, Soriano TL, Carandang E, Hermawan 2012. Beyond compliance: project on an integrated systems approach for pest risk management in South East Asia. Bulletin OEPP/EPPO 42(1): 109-116.

Philibert A, Desprez-Loustau M-L, Fabre B, Frey P, Halkett F, Husson C, Lung-Escarmant B, Marcais B, Robin C, Vacher C, Makowski D 2011. Predicting invasion success of forest pathogenic fungi from species traits. Journal of Applied Ecology 48: 1381-1390.

Quinlan M, Ikin R 2009. A review of the application of Systems Approach to risk management in plant health. EU Framework 7 Research Project Report No. 212459, Deliverable No. 4.2. 69 p.

Rodil IF, Compton TJ, Lastra M 2012. Exploring macroinvertebrate species distributions at regional and local scales across a sandy beach geographic continuum. PLoS ONE 7(6): e39609. http://www.plosone.org/ article/info\%3Adoi\%2F10.1371\%2Fjournal. pone.0039609 (accessed 6 June 2013).
Schmidt JP, Drake JM 2011. Time since introduction, seed mass, and genome size predict successful invaders among the cultivated vascular plants of Hawaii. PLoS ONE 6(3): e17391. http://www.plosone.org/ article/info\%3Adoi\%2F10.1371\%2Fjournal. pone.0017391 (accessed 6 June 2013).

Speirs-Bridge A, Flander L, Robinson A 2010. Expert elicitation using real-time virtual panels. Australian Centre of Excellence for Risk Analysis. http://www.acera.unimelb.edu. $\mathrm{au} / \mathrm{sra} / 2010 /$ Presentations/Speirs-Bridge.pdf (accessed 15 May 2013).

Sugiyama S 2008. Monte Carlo simulation / risk analysis on a spreadsheet: Review of three software packages. Foresight (9): 36 - 39.

Waage JK, Mumford JD 2008. Agricultural biosecurity. Philosophical Transactions of The Royal Society Biological Sciences 363: 863-876.

Walshe T, Burgman M. 2010. A framework for assessing and managing risks posed by emerging diseases. Risk Analysis 30 (2): 236-249.

Whittle P, Johnson S, Leach A, Holt J, Quinlan M, Mengersen K, Mumford J 2012. Developing systems approaches for phytosanitary pest risk management using Control PointBayesian networks. Australian Bayes Nets Model Society.http://abnms.org/conferences/ abnms2012/program.php (accessed 15 May 2013).

Witten IH, Frank E, Hall MA 2011. Data mining: practical machine learning tools and techniques. Morgan Kauffman Publishers, Burlington, Massachusetts, USA. ISBN: 9780-12-374856-0.

Yamamura K, Katsumata H 1999. Estimation of the probability of insect pest introduction through imported quantities. Researches on Population Ecology 41: 275-282. 